
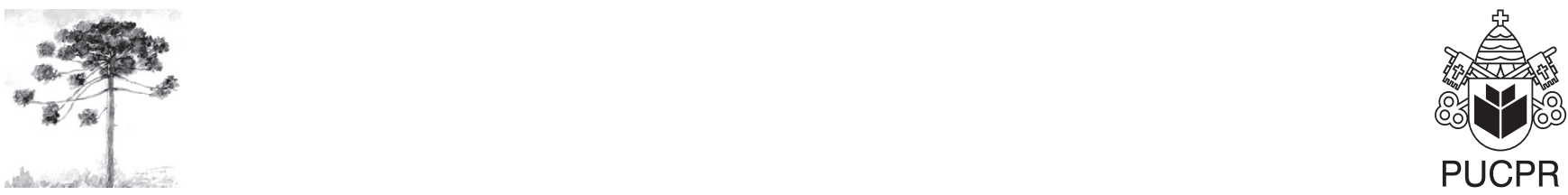

PUCPR

\title{
Homeopatia no controle do Trypanosoma evansi em ratos infectados experimentalmente
}

\author{
Homeopathy in the control of Trypanosoma evansi in \\ experimentally infected rats
}

\author{
Aleksandro Schafer da Silva ${ }^{[a]}$, Camila Belmonte Oliveira ${ }^{[a]}$, \\ Régis Adriel Zanette ${ }^{[b]}$, Ivia Gonzalez Monteiro ${ }^{[\mathrm{c}]}$
}

[a] Pós-Graduação em Medicina Veterinária da Universidade Federal de Santa Maria (UFSM), Santa Maria - RS, Brasil.

[b] Pós-Graduação em Ciências Veterinária da Universidade Federal do Rio Grande do Sul (UFRGS), Porto Alegre - RS, Brasil.

[c] Departamento de Microbiologia e Parasitologia da Universidade Federal de Santa Maria (UFSM), Santa Maria - RS, Brasil, e-mail: sgmonteiro@uol.com.br

\section{Resumo}

O objetivo deste estudo foi avaliar a eficácia de uma solução homeopática no controle de infecções por Trypanosoma evansi em ratos infectados experimentalmente. Para o trabalho foram utilizados 24 ratos machos (Rattus norvergicus), divididos em quatro grupos (A, B, C, D), com seis animais cada. Todos os animais foram infectados via oral com uma dose de $1,2 \times 10^{5}$ tripanosomas/animal no dia zero. O grupo A, grupo controle, recebeu somente água. Os grupos tratamento $(\mathrm{B}, \mathrm{C}, \mathrm{D})$ receberam diariamente o produto homeopático diluídos em água, na dose recomendada pelo fabricante. No grupo B a solução foi administrada por 30 dias antes da inoculação do T. evansi, sendo mantida até o fim do experimento. No grupo $\mathrm{C}$ a terapia homeopática iniciou no dia da inoculação (dia zero). Para os roedores do grupo D o tratamento iniciou-se 20 dias após inoculação, quando foram detectados os flagelados no esfregaço sanguíneo. Não houve diferença estatística no período pré-patente, longevidade e ganho de peso dos ratos tratados em comparação ao grupo controle. Com base nos dados, conclui-se que o produto homeopático utilizado nas doses testadas não controlou a infecção por T. evansi em $R$. norvergicus.

Palavras-chave: Trypanosoma evansi. Rattus norvergicus. Homeopatia. 


\begin{abstract}
The aim of this research study was to evaluate the efficacy of a homeopathic solution in the control of infections by Trypanosoma evansi in experimentally infected rats. Twenty four male rats (Rattus norvergicus) divided in four equal groups $(A, B, C, D)$ were used. All animals were infected with an oral dose of $1.2 \times 10^{5}$ trypanosomes in the day zero. Animals from group $A$ (control group) received only water. Groups $B, C$ and $D$ received the indicated dose of the homeopathic product daily. Daily medications started 30 days before day zero for group B, at day zero for group $C$ and 20 days after day zero for group $D$, when flagellate forms of $T$. evansi were detected in the blood smears. No statistical differences in prepatent period, longevity and weigh gain were observed between treated and non-treated groups. Based upon the results it's concluded that the homeopathic product in the evaluated doses was not efficient in the control of the infection by $\mathrm{T}$. evansi in $\mathrm{R}$. norvergicus.
\end{abstract}

Keywords: Trypanosoma evansi. Rattus norvergicus. Homeopathy.

\section{Introduçáo}

Tripanossomoses são doenças provocadas por protozoários patogênicos do gênero Trypanosoma, que têm larga distribuição e importância econômica na África, Ásia e América latina (1, 2). O Trypanosoma evansi é um flagelado da seção salivaria, causador da doença secularmente conhecida como mal de cadeiras em equinos no Pantanal mato-grossense (3). No Brasil, T. evansi afeta principalmente equinos, e a prevalência da infecção varia de região para região (4, 5). No entanto, este parasita pode causar doença em cães (5-7), capivaras (Hydrochaeris hydrochaeris), quatis (Nasua nasua), bovinos, búfalos e tatus (Dasypus sp.) (5) e homem (8).

A homeopatia fundamenta-se em princípios distintos da medicina convencional, aplicando o princípio de cura pela similitude, por meio de substâncias previamente experimentadas em indivíduos sadios, em doses infinitesimais. A aplicação terapêutica desses pressupostos valoriza a individualidade humana, elegendo, dentre as milhares de substâncias experimentadas, aquela que engloba a totalidade de sintomas característicos de cada paciente (nos aspectos psíquicos, emocionais, gerais e clínicos), empregando, para um mesmo tipo de doença, medicamentos distintos para cada indivíduo enfermo (9). A singularidade de conceitos e aplicações, explicada ou entendida de forma distorcida, tem gerado dissensões seculares entre médicos homeopatas e não homeopatas, criando uma segunda barreira nas tentativas de aproximação dessas racionalidades distintas (10).

Alternativas para o controle da tripanossomose são de fundamental importância, pois os medicamentos não têm mantido os animais livres da parasitemia, ocorrendo recidiva da doença em muitos casos. O objetivo deste trabalho foi avaliar a eficácia de um produto homeopático no controle de infecções por T. evansi em ratos.

\section{Material e métodos}

No decorrer do experimento foram utilizados 24 ratos (Rattus norvergicus), machos com 2 meses de idade. Esses roedores foram divididos em quatro grupos de seis animais cada, sendo estes pesados no inicio e fim do experimento. Os animais tiveram um período de adaptação de 15 dias quando receberam antihelmíntico à base de fenbendazole diluído em água.

Os animais foram infectados via oral (11), com uma cepa de T. evansi mantida em nitrogênio, desde seu isolamento de cão naturalmente infectado do município de Uruguaiana, RS (12). A dose administrada foi de $1,2 \times 10^{5}$ tripanosomas por rato. A inoculação de todos os grupos foi realizada no dia zero.

Os grupos formados receberam a mesma solução homeopática $\left(\mathrm{CH}-\mathrm{Coxvit}^{\circledR}\right)$ à base de Allium sativum, Carduus marianus e Arsenicum album, em três diferentes períodos, a fim de avaliar a ação profilática e curativa do produto. O grupo A foi utilizado como grupo controle, não recebeu nenhuma medicação com a água. Os grupos tratamento $(\mathrm{B}, \mathrm{C}, \mathrm{D})$ receberam diariamente o produto homeopático diluídos em água, na dose recomendada pelo fabricante. No grupo B a solução foi administrada por 30 dias antes da inoculação do T. evansi, e foi mantida até o fim 
do experimento. No grupo C a terapia homeopática teve início no dia da inoculação (dia zero). Para os roedores do grupo D o tratamento iniciou-se 20 dias após inoculação, quando foram detectados os flagelados no esfregaço sanguíneo. A dose diária utilizada na terapia foi de 100 gotas por litro de água, conforme recomendação do fabricante.

Após inoculação dos parasitos, os animais foram avaliados diariamente, por meio da pesquisa microscópica de esfregaço do sanguíneo periférico (Figura 1) (13). As lâminas confeccionadas foram examinadas em aumento de 1.000 vezes. Para considerar eficaz o produto homeopático, avaliou-se sua capacidade em reduzir ou eliminar o parasito da circulação dos ratos. A análise estatística dos dados foi feita por meio da análise de variância (ANOVA), seguida por aplicação do teste de Tukey para comparação entre as médias de período pré-patente, longevidade e ganho de peso dos ratos em cada tratamento (14).

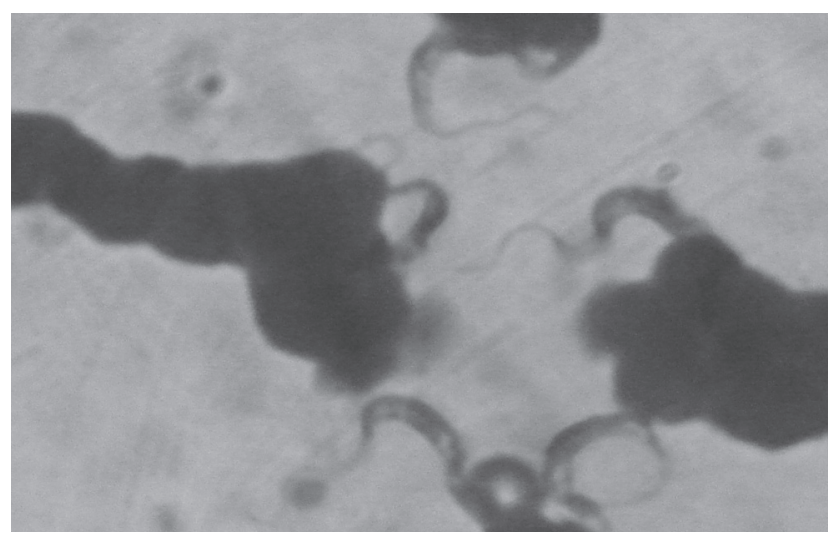

Figura 1 - Trypanosoma evansi obtido de esfrega sanguíneo de ratos em aumento de 1.000 vezes, corado em lâmina com Panótico Rápido

\section{Resultados}

A terapia homeopática administrada aos animais não foi eficaz no controle da infecção por $T$. evansi, pois todos os ratos do experimento morreram em decorrência da doença. O período pré-patente, a longevidade e o ganho de peso dos animais não apresentaram diferença estatística significativa. No entanto, os $R$. norvergicus dos grupos B e $\mathrm{C}$ apresentaram longevidade superior a dos grupos A e D (Tabela 1).

\section{Discussão e conclusão}

A longevidade dos ratos deste estudo foi semelhante à observada por pesquisadores (15) que testaram três drogas no controle da infecção experimental em ratos com T. evansi. $\mathrm{O}$ aceturato de diminazeno foi o único que diferiu dos medicamentos testados e do produto homeopático avaliado no presente trabalho quanto à longevidade dos animais após terapia, os quais sobreviveram em média 43 dias (15).

O estado corporal dos ratos foi idêntico para todos os tratamentos, mostrando não haver diferença estatística entre o ganho de peso dos animais tratados e grupo controle. Em outro trabalho, quando se utilizou a mesma solução homeopática testada neste estudo para o tratamento de coccídeos em ratos e camundongos, também não foi verificada diferença no peso dos animais (16).

A solução homeopática testada não teve efeito curativo para os ratos infectados com T. evansi (Tabela 1). Conforme a literatura, já foi verificada atividade antiparasitária in vitro da allicina, principal

Tabela 1 - Média e desvio padrão do período pré-patente, longevidade e ganho de peso de ratos infectados por Trypanosoma evansi, submetidos ao tratamento homeopático

\begin{tabular}{lcccc}
\hline Produto & Grupo & $\begin{array}{c}\text { Período pré-patente } \\
\text { (dias) }\end{array}$ & $\begin{array}{c}\text { Longevidade } \\
\text { (dias) }\end{array}$ & $\begin{array}{c}\text { Ganho de peso } \\
\text { (gramas) }\end{array}$ \\
\hline Controle & A & $18,6( \pm 3,2)$ & $5,8( \pm 0,7)$ & $55,0( \pm 9,2)$ \\
& B & $18,5( \pm 2,8)$ & $9,0( \pm 1,9)$ & $62,2( \pm 13,4)$ \\
Homeopático & C & $17,6( \pm 3,2)$ & $8,1( \pm 1,5)$ & $53,9( \pm 10,6)$ \\
& D & $19,6( \pm 3,7)$ & $6,1( \pm 0,7)$ & $56,4( \pm 7,8)$ \\
\hline
\end{tabular}

Nota: As médias não diferiram estatisticamente entre si a 5\% de probabilidade do teste de Tukey. 
constituinte do alho (A. sativum), em protozoários e parasitos do homem e de animais. Esta foi eficaz contra Entamoeba histolitica, Ascaris lumbricoides, Giardia lambia, Trypanosoma brucei brucei, T. rhodisiense, T. gambiense, T. evansi, T. congolense e T. equiperdum (17-20), mas no presente estudo a solução testada, que continha allicina, não teve eficácia, pois todos os animais morreram. Entretanto, acredita-se que este produto tenha alguma influência na profilaxia, sobre a parasitemia de ratos infectados por T. evansi, pois os ratos dos grupos $\mathrm{B}$ e $\mathrm{C}$ tiveram longevidade superior aos dos grupos A e D (Tabela 1).

Com base nos resultados apresentados neste estudo, conclui-se que a homeopatia na dose testada não controlou a infecção por T. evansi em $R$. norvergicus. A solução homeopática não interferiu no ganho de peso dos animais.

\section{Agradecimentos}

Agradecemos a colaboração, para realização desta pesquisa, da médica veterinária homeopata Angélika Sharom pela doação do produto homeopático utilizado neste estudo.

\section{Referências}

1. Gardiner PR. Recent studies of the biology of Trypanosoma vivax. Adv Parasitol. 1989;28:229-317.

2. Lun ZR, Desser SS. Is the broad range of hosts and geographical distribuition of Trypanosoma evansi attributable to the loss of maxicircle kinetoplast DNA? Parasitol Today. 1995;11(4):131-3.

3. Silva RAMS, Seidl A, Ramirez L, Dávila AMR. Trypanosoma evansi e Trypanosoma vivax - Biologia, Diagnóstico e Controle. Corumbá: Embrapa Pantanal; 2002.

4. Dávila AMR, Silva RAMS. Animal trypanosomiasis in South America. Current status, partnership, and information technology. Ann N Y Acad Sci. 2000; 916:199-212.

5. Herrera HM, Davila AM, Norek A, Abreu UG, Souza SS, Andrea PS, et al. Enzootiology of Trypanosoma evansi in Pantanal, Brazil. Vet Parasitol. 2004;125(3-4):263-75.
6. Nunes VLB, Oshiro ET, Dorval MEC, Espíndola MA, Cristaldo G, Rocha HC, et al. Estudos epidemiológicos sobre leishmaniose tegumentar (LT) e mal das cadeiras no município de Corguinho, Mato Grosso do Sul - Estudo de reservatórios, 1992-1994. Rev Bras Parasitol Vet. 1994;3:29-35.

7. Silva RAMS, Herrera HM, Domingos LBS, Ximenes FA, Dávila AMR. Pathogenesis of Trypanosoma evansi infection in dogs and horses: hematological and clinical aspects. Ciência Rural. 1995;25:233-8.

8. Joshi PP, Shegokar VR, Powar RM, Herder S, Katti R, Salkar HR. Human Trypanosomosis caused by Trypanosoma evansi in India: the first case report. Am J Trop Med Hygiene. 2005;73(3):491-5.

9. Teixeira MZ. Panorama da pesquisa em homeopatia: iniciativas, dificuldades e propostas. Diagn Tratamento. 2004;9(3):98-104.

10. Luz MT. A arte de curar versus a ciência das doenças: história social da homeopatia no Brasil. São Paulo: Dynamis; 1996.

11. Silva AS, Ceolin LV, Oliveira CB, Doyle RL, Monteiro SG. Infecção via oral por Trypanosoma evansi em animais de laboratório. Ciência Rural. 2007;37(3):897-900.

12. Colpo CB, Monteiro SG, Stainki DR, Colpo TB, Henriques GB. Infecção natural por Trypanosoma evansi em cão no Rio Grande do Sul. Ciência Rural. 2005;35(3):717-9.

13. Silva AS, Doyle RL, Monteiro SG. Métodos de contenção e confecção de esfregaço sanguíneo para pesquisa de hemoparasitas em ratos e camundongos. Revista da Faculdade de Zootecnia, Veterinária e Agronomia. 2006;13(2):83-7.

14. Silva FAZ, Azevedo CAV. Versão do programa computacional Assistat para o sistema operacional Windows. Rev Bras Prod Agroindustriais. 2002;4(1):71-8.

15. Doyle RL, Silva AS, Santurio JM, Monteiro SG, Graça DL. Eficácia de medicamentos no controle da infecção experimental por Trypanosoma evansi em ratos. Acta Scientiae Veterinariae. 2007;35(1):46-9.

16. Silva AS, Soares CDM, Coradini GP, Zanette RA, Oliveira CB, Sharom A, Monteiro. Homeopatia na terapia de animais de laboratório naturalmente infectados por coccídeos. Estud Biol. 2007;29(67):145-9. 
17. Kalyesa R. Screening of indigenous plants for antihelminthic action against humam Ascaris lumbricoides. Ind J Physiol Pharmacol. 1975;19(1):47-9.

18. Blasi V. Amoebicidal effect of essencial oils in vitro. J Toxicol Clin Exp. 1990;10(6):361-73.

19. Lun ZR, Burri C, Menzinger M, Kaminsky R. Antiparasitic activity of diallyl trisulfide (dasuansu) on human and animal pathogenic protozoa (Trypanosoma sp., Entamoeba histolytica and Giardia lambria) in vitro. Ann Soc Belg Med Trop. 1994;74(1):51-9.
20. Ankri S, Mirelman D. Antimicrobial properties of allicin from garlic. Microbes Infect. 1999;1(2):125-9.

Recebido: 03/05/2009

Received: 05/03/2009

Aprovado: 12/08/2009

Approved: 08/12/2009 